\title{
Risk factors for early sudden deaths and severe apparent life-threatening events
}

\author{
Anette Poets, Michael S Urschitz, Renate Steinfeldt, Christian F Poets
}

Department of Neonatology, Tuebingen University Hospital, Tuebingen, Germany

\section{Correspondence to}

Christian F Poets, Department of Neonatology, Tuebingen University Hospital, Calwerstr 7, 72076 Tuebingen, Germany; christian-f.poets@med. uni-tuebingen.de

Received 1 August 2011 Accepted 5 January 2012 Published Online First 31 January 2012
ABSTRACT

Objective To identify potential risk factors for unexpected sudden infant deaths (SID) and severe apparent life-threatening events (S-ALTE) within $24 \mathrm{~h}$ of birth.

Design Case-control study embedded in an epidemiological survey over a 2-year period.

Patients and methods Throughout 2009, every paediatric department in Germany was asked to report cases of unexplained SID or S-ALTE in term infants with a 10-min Apgar score $\geq 8$ to the Surveillance Unit for Rare Pediatric Conditions. Throughout 2010, the inclusion criteria were extended to infants $\geq 35$ week gestational age and those where an explanation for the deterioration had been found. For each unexplained case, hospitals were asked to fill in a questionnaire for 3 (near-)term controls with good postnatal adaptation at the age (in minutes) when the event had occurred in the case under study.

Results Of the 85 cases reported, 34 fulfilled the entry criteria; of these, two were near-term newborns and, in three cases, a cause had been identified for the event. For the 31 cases with unknown cause for the event (13 males; mean (SD) gestational age 38.9 (1.7) week), the authors gathered 93 controls (51 male infants; 38.9 (1.4) week). As significant risk factors for S-ALTE and SID, the authors could identify primipara (OR 6.22; 95\% Cl 2.11 to 18.32 ) and potentially asphyxiating position (OR 6.45; $95 \% \mathrm{Cl} 1.22$ to 34.10 ).

Conclusions Close observation of newborns seems necessary, particularly in primipara; a potentially asphyxiating position should be avoided.

\section{INTRODUCTION}

In Germany, in 2009, we determined the incidence of unexpected sudden infant deaths (SID) and severe apparent life-threatening events (S-ALTE) in term infants ( $\geq 37$ weeks' gestational age) within $24 \mathrm{~h}$ of birth after a good postnatal adaptation (10-min Apgar score $\geq 8) .{ }^{1}$ Seventeen cases met inclusion criteria, corresponding to an incidence of 2.6 per 100000 live births.

It was noticeable that more than half of the events occurred within 2 h of birth (mostly after vaginal delivery) and more than two-thirds of affected newborns were lying on the breast or abdomen of their mother or near to and facing her. It was also striking that most mothers were primipara, and that, in 7 of 17 cases, the mother had not recognised the deterioration of her baby despite her being present and awake.

In 2010, we continued the survey. As risk factors remain speculative without adequate controls, our aim was to obtain information on birth

\section{What's known on this subject}

- The incidence proportion of sudden infant death and severe apparent life-threatening events (ALTE) in term newborns within $24 \mathrm{~h}$ of birth is about 2.6 per 100000 live births in Germany.

- Primipara, postnatal fatigue and an asphyxiating position have been proposed as risk factors.

\section{What this study adds}

Primipara and an asphyxiating position are indeed risk factors for sudden infant death and severe ALTE in term newborns within $24 \mathrm{~h}$ of birth

characteristics and postnatal practices for infants born in hospitals reporting a case, and to compare cases and controls concerning putative risk factors.

\section{METHODS}

As part of the Surveillance Unit for Rare Pediatric Conditions in Germany, ${ }^{2}$ all paediatric departments in Germany were sent monthly reporting cards between 1 January 2009 and 31 December 2009 asking them to notify the study centre of any case of unexplained SID or S-ALTE occurring within $24 \mathrm{~h}$ of birth in a full-term infant ( $\geq 37$ week gestational age) after a good postnatal adaptation (10-min Apgar score 28 ). S-ALTE was defined as an acute state of cyanosis or pallor and unconsciousness, which was felt to require bagging, or intubation with or without cardiac compressions.

We continued the study in 2010 and extended the entry criteria to include near-term infants $(\geq 35$ week gestational age) and those where an explanation for the deterioration had been found in retrospect. We made this change because, during the first year of our study, we had been notified of four near-term infants who met inclusion criteria except for their premature birth and, also, of three newborns for whom an explanation for the event had been discovered later.

Hospitals reporting a case received a questionnaire covering the birth, the postnatal situation and the circumstances of the event. In addition, we asked for the infant's hospital discharge letter, and for the autopsy protocol in the case of 
Table 1 Comparison between cases and controls concerning possible risk factors for life-threatening events (only events without an identified cause are included)

\begin{tabular}{|c|c|c|c|c|c|}
\hline Suspected risk factor & Cases $(n=31)$ & Controls $(n=93)$ & p Value & $\mathbf{O R}$ & $95 \% \mathrm{CI}$ \\
\hline Potentially asphyxiating position* & $25 / 29(86 \%)$ & $60 / 90(67 \%)$ & 0.028 & 6.45 & 1.22 to 34.10 \\
\hline Primipara & $25 / 31(81 \%)$ & $44 / 93(47 \%)$ & 0.001 & 6.22 & 2.11 to 18.32 \\
\hline Vaginal delivery & $26 / 31(84 \%)$ & $68 / 93(73 \%)$ & 0.179 & 2.22 & 0.69 to 7.09 \\
\hline $\begin{array}{l}\text { Maternal use of sedatives in } 24 \mathrm{~h} \\
\text { before delivery }\end{array}$ & $6 / 28(21 \%)$ & $17 / 93(18 \%)$ & 0.606 & 1.42 & 0.37 to 5.42 \\
\hline
\end{tabular}

*Infant lying on mother's breast/abdomen or near to and facing her.

†Not all mothers had initiated breastfeeding at this point.

SID; all documents had to be anonymised before sending them to us. Having collected these data, we excluded cases not meeting inclusion criteria (eg, those who had only been stimulated).

Applying a matched pairs design, we asked every hospital reporting an unexplained case to fill in anonymised questionnaires for three (near-)term infants with a 10-min Apgar score $\geq 8$ born within a few days after receipt of the questionnaires. These had to be filled in at the age (in min) at which the deterioration of the case had been recognised. Questions were on parity, mode of delivery, maternal use of sedatives in the past $24 \mathrm{~h}$ before delivery, whether the mother was awake or asleep, infants' sex, sleeping position and sucking behaviour.

In comparing the information for cases and controls, we tried to verify putative risk factors for such events: primipara, a strenuous delivery, mother not supervising the infant because of being asleep or sedated and a potentially asphyxiating position of the infant, defined as lying prone on the breast or abdomen of its mother or close to her in a side position. As some health professionals reported that the cases had sucked excessively while being breastfed just before the event occurred, we also wanted to investigate the relationship between excessive sucking and such events, despite the lack of a clear definition for 'excessive sucking'. Because of the predominance of the male sex in SID cases in the first year of life, ${ }^{3}$ we also wanted to check the data for each infant's gender.

The study protocol, including an informed consent waiver, was approved by the Ethics Committee of Tuebingen University Hospital.

\section{Statistical analysis}

Descriptive statistics (numbers and percentages, means and SDs or medians and ranges) were used to summarise subject characteristics wherever appropriate. According to study design, unadjusted OR and their 95\% CI were calculated using unconditional binary logistic regression analysis. The case-control status of the individual (ie, case or control) was used as dependent variable. The following risk factors were investigated for their association with the case-control status: primipara, mode of delivery, maternal use of sedatives in the last $24 \mathrm{~h}$ before delivery, sleeping mother, potentially asphyxiating position of the newborn, excessive sucking and gender. Due to the small sample size, multiple regression models to adjust for confounding were not built. A p value of $<0.05$ was considered statistically significant. All analyses were carried out with statistical software (IBM SPSS Statistics version 19).

\section{RESULTS}

Children's hospitals notified us of 85 cases that had occurred in 2009 and 2010; in 2009, 17 of these had met inclusion criteria. In 2010, 17 additional infants met inclusion criteria; two of them were near-term infants, and in three, an explanation was found at a later stage (pulmonary hypertension of the newborn, pneumonia and a Norovirus infection, respectively). In 2010, we were notified of one additional event that had occurred at home; in this case we could not obtain detailed information about the circumstances of the event.

For each of the 31 unexplained cases of SID or S-ALTE (13 males; mean (SD) gestational age 38.9 (1.7) week), we received information on three controls (51 males; 38.9 (1.4) week). A comparison between cases and controls concerning possible risk factors for events is provided in table 1.

The median (25/75 percentile) age at which the case infant was found collapsed (the situation described for controls) was $90(40 / 360) \mathrm{min}$ in cases and $90(59 / 285) \mathrm{min}$ in controls.

\section{DISCUSSION}

The number of events reported to us in 2010 was comparable with that identified during the first year of this study. ${ }^{1}$ In a recently published nationwide survey in the UK, which was very similar to our study design, the incidence was slightly higher. ${ }^{4}$ This could be related to a better reporting of cases or to a less-intense postnatal surveillance, leading to a higher number of events.

In comparing cases with controls, we could confirm 'asphyxiating position' and 'primipara' as potential risk factors for SID or S-ALTE within $24 \mathrm{~h}$ of birth. While a potentially asphyxiating position is a well-known risk factor for SID in general ${ }^{3}$ and also supposed as a risk factor for such events in the first $24 \mathrm{~h}$ of life ${ }^{5-7}$ primipara has only been reported as a risk factor for early SID. ${ }^{5-7}$ Beyond the first day of life, the risk for SID seems to increase with parity. ${ }^{8}$ In our previous publication, ${ }^{1}$ we proposed some explanations for why the above factors may predispose a term newborn to life-threatening events: 'primipara' because of an assumed inexperience of the mother in assessing her newborn baby and surveying it, and 'asphyxiating position' because of the risk of upper airway obstruction in a newborn lying prone on, or very near to, its mother. In this regard, a contributing factor could be maternal obesity; unfortunately, we did not obtain information on maternal body mass index. Another factor possibly leading to a dangerous situation for the infant could be maternal exhaustion or sedation around delivery. There was no difference in maternal use of sedatives, and labour duration could not be compared between cases and controls as more cases were born to primipara, leading to a 
longer average labour duration in this group, and because we did not match cases and controls for mode of delivery.

In discussions of life-threatening events having occurred in the delivery room, health professionals have occasionally mentioned that they had noticed an excessive sucking of the infant shortly before the event had occurred. Therefore, we compared the frequency of excessive sucking in cases and controls but, contrary to our hypothesis, this factor was significantly more prevalent in controls. This might point to control infants being more vigorous, but may also simply be the result of recall bias, which inevitably is a caveat of our study anyway.

We did not analyse smoking as a potential risk factor for early SID/S-ALTE as the prevalence of smoking in cases (4\%) was too low to be plausible, which may be related to the fact that this information was only collected several weeks after the event had occurred.

In 2010, we were notified of three cases where a preventable cause for the infant's deterioration had been identified (although not asked for in 2009, the same number had been reported during that year).

The main limitation of this study is the relatively small number of cases and controls and, hence, the lack of statistical power. Some putative risk factors with elevated but non-significant OR (eg, mother asleep) could become significant in future larger studies. Another limitation was the selection of controls, which was left to the discretion of the hospital staff, leaving potential for selection bias. Although most questionnaires were filled in at the age demanded (ie, when the case infant had been found collapsed), this was not done exactly for every control.

\section{CONCLUSION}

When a newborn is lying on its mother's breast/abdomen, or near to and facing her, nurses and midwives should be aware of the increased possibility that a life-threatening event might occur. This is especially the situation with a mother who is primipara. It is necessary to check on these newborns at regular, short intervals and to pay particular attention to confirming that breathing is unobstructed.

Contributors Anette Poets was involved in the study design, supervised the data collection and analysis and wrote the first draft of the manuscript; Michael S Urschitz was responsible for statistical analysis. Renate Steinfeldt was involved in designing the study and data collection; Christian F Poets initiated and supervised the study and revised the manuscript. Anette Poets had full access to all of the data in the study and takes responsibility for the integrity of the data and the accuracy of the data analysis.

Acknowledgements The authors are most grateful to the physicians, midwives and nurses who have helped so much by taking part in this study, filling in our questionnaires and reporting their findings to us, and to Derek Stebbens, M A, for improving the language of this manuscript.

Funding Beitlich Foundation.

Competing interests None.

Ethics approval Ethics Committee of Tuebingen University Hospital.

Provenance and peer review Not commissioned; externally peer reviewed.

Data sharing statement No additional data.

\section{REFERENCES}

1. Poets A, Steinfeldt R, Poets CF. Sudden deaths and severe apparent life-threatening events in term infants within 24 hours of birth. Pediatrics 2011;127:e869-73.

2. Göbel U, Heinrich B, Krauth KA, et al. (Process and outcome quality of the German Paediatric Surveillance Unit (ESPED)). Klin Padiatr 2010;222:92-7.

3. Carpenter RG, Irgens LM, Blair PS, et al. Sudden unexplained infant death in 20 regions in Europe: case control study. Lancet 2004;363:185-91.

4. Becher JC, Bhushan SS, Lyon AJ. Unexpected collapse in apparently healthy newborns - a prospective national study of a missing cohort of neonatal deaths and near-death events. Arch Dis Child Fetal Neonatal Ed 2012;97:F30-4.

5. Gatti $\mathbf{H}$, Castel C, Andrini P, et al. (Cardiorespiratory arrest in full term newborn infants: six case reports). Arch Pediatr 2004;11:432-5.

6. Peters C, Becher JC, Lyon AJ, et al. Who is blaming the baby? Arch Dis Child Fetal Neonatal Ed 2009;94:F377-8.

7. Dageville C, Pignol J, De Smet S. Very early neonatal apparent life-threatening events and sudden unexpected deaths: incidence and risk factors. Acta Paediatr 2008;97:866-9.

8. Alm B, Norvenius SG, Wennergren G, et al. Changes in the epidemiology of sudden infant death syndrome in Sweden 1973-1996. Arch Dis Child 2001;84:24-30. 